\title{
TAGUNG
}

\section{Europäisierte Regulierungsstrukturen und -netzwerke: Basis einer künftigen Infrastrukturvorsorge?}

\author{
Cornelia Manger-Nestler und Gregor Noack*
}

Europäisierte Regulierungsprozesse als juristische und ökonomische Herausforderung

Die Regulierung von Märkten und das Management von Risiken werden in immer stärkerem Maße durch Organe und Institutionen der Europäischen Union bestimmt. Andererseits kann rasanten technischen Entwicklungen und krisenhaften ökonomischen Prozessen einer immer enger vernetzten Wirtschaft wie Gesellschaft mit den Regelungsmechanismen des klassischen Nationalstaates kaum mehr wirkungsvoll entgegengetreten werden. Selbst supranationale Gremien beziehungsweise auf Freiwilligkeit beruhende transnationale Netzwerke tun sich schwer, diese Erscheinungen einzufangen. Und schließlich ist der Begriff , Regulierung" schon in seinem nationalrechtlichen Kern einem ständigen Wandel unterworfen; in Randbereichen verliert er weiter an Trennschärfe. Während der bisherige Diskurs geprägt wurde von spezifischen Rechtsfragen ausgewählter Sektoren, sollte diese Konferenz den schillernden Begriff eines ,europäischen' beziehungsweise ,europäisierten" Regulierungsrechts in zentralen Facetten stärker konturieren, sektorübergreifende Strukturen aufzeigen und das Thema somit einer größeren Systematisierung im europäischen Kontext zuführen. Zugleich sollten Vielfalt und Breite des Regulierungsrechts an sechs ausgewählten ,Regulierungsfeldern erläutert werden: Finanz-, Wasser-, Telekommunikations-, Post-, Verkehrs- und Energiemärkte.

\section{Europäisierte Regulierungsstruk- turen und -netzwerke als Basis einer künftigen Infrastrukturvorsorge}

Mit freundlicher Unterstützung des Arbeitskreises Europäische Integration e.V. und der Europäischen Kommission

Leipzig, 5.-6. Mai 2011

Sektorübergreifende Fragen des Regulierungsrechts

Begrüßung

Prof. Dr. Cornelia MANGER-NESTLER, LL.M., Professur für Deutsches und Internationales Wirtschaftsrecht, Hochschule für Technik, Wirtschaft und Kultur, Leipzig

\section{Moderation}

Prof. Dr. Josef RUTHIG, Lehrstuhl für Öffentliches Recht, Europarecht, Rechtsvergleichung, Johannes-Gutenberg-Universität Mainz

Warum und wozu Regulierung im europäischen Mehr-Ebenen-System? - Gründe für bzw. Ziele von Regulierung

Prof. Dr. Günter KNIEPS, Direktor des Instituts für Verkehrswissenschaft und Regionalpolitik, Universität Freiburg im Breisgau

Die politische Unabhängigkeit der Bundesnetzagentur Prof. Dr. Bernd HOLZNAGEL, Institut für Informations-, Telekommunikations- und Medienrecht, Westfälische Wilhelms-Universität Münster

Organisatorisch-prozedurale Aspekte sektorübergreifender Regulierungsstrukturen und-instrumente Dr. Iris HENSELER-UNGER, Vizepräsidentin der Bundesnetzagentur, Bonn

* Prof. Dr. iur. Cornelia Manger-Nestler, LL.M., Inhaberin der Professur für Deutsches und Internationales Wirtschaftsrecht an der Hochschule für Technik, Wirtschaft und Kultur, Leipzig.

Dipl.-Jur. Gregor Noack, wissenschaftlicher Mitarbeiter an der Professur für Deutsches und Internationales Wirtschaftsrecht an der Hochschule für Technik, Wirtschaft und Kultur, Leipzig. 
Insgesamt bot die von Ludwig Gramlich und Cornelia Manger-Nestler geleitete Tagung Juristen wie Ökonomen ein sinnstiftendes Forum und führte $\mathrm{zu}$ einem gewinnbringenden Diskurs von nationalen und europäischen Vertretern aus Wissenschaft, Politik und Regulierungspraxis.

\section{Grundfragen sektorübergreifender Regulie- rungsstrukturen}

Ziel des von Josef Ruthig moderierten ersten Panels war es, allgemeine, das heißt sektorübergreifende Fragen des Regulierungsrechts zu erörtern; dazu zählten Ziele von Regulierung, organisatorisch-institutionelle Aspekte (Akteure), Regulierungsinstrumente und -befugnisse sowie Rechtsschutzfragen.

Aus ökonomischer Sicht stellte Günter Knieps als Gründe für Regulierung die Kompatibilität und das Management von gemeinsam genutzten knappen Ressourcen (öffentliche Güter), die flächendeckende Versorgung zum sozial erwünschten Tarif sowie die Gewährleistung von funktionsfähigem Wettbewerb heraus. Neben den Anforderungen an eine Anreizregulierung wurden Umfang sowie Reformpotenziale von Regulierungsinstrumenten im europäischen Mehrebenensystem, insbesondere ein disaggregiertes Regulierungsmandat näher beleuchtet.

Der politischen Unabhängigkeit der Bundesnetzagentur (BNetzA) im Spannungsfeld zu demokratischen Grundsätzen widmete sich Bernd Holznagel. Als selbstständige Bundesoberbehörde ist die BNetzA grundsätzlich weisungsgebunden. Dem entgegen stehen nun die in den neuen ,Binnenmarktpaketen ' enthaltenen europäischen Vorgaben ${ }^{1}$, wonach die Nationale Regulierungsbehörde (NRB) bei der Wahrnehmung ihrer Aufgaben weder Weisungen von Regierungsstellen einholt noch entgegennimmt. Geprüft wurde diese se-
Regulierungsinstrumente und Befugnisse bei der Regulierung marktbeherrschender Unternehmen Dr. Ulrich STUMPF, Direktor Regulierung und Wettbewerb, Wissenschaftliches Institut für Infrastruktur und Kommunikationsdienste, Bad Honnef

Entscheidungsprärogativen der nationalen Regulierungsbehörde und gerichtliche Kontrolldichte Dr. Kurt GRAULICH, Richter am Bundesverwaltungsgericht, Leipzig

Strukturen des Rechtsschutzes gegen Maßnahmen von Regulierungsbehörden - Gemeinschaftsrechtlicher Rahmen und nationalstaatliche Ausgestaltung RA Prof. Dr. Joachim SCHERER, LL.M., Baker \& McKenzie, Frankfurt am Main

Rechtsschutz für Regulierung in Österreich und in Europa

Prof. Dr. Stefan STORR, Institut für Österreichisches, Europäisches und Vergleichendes Öffentliches Recht, Politikwissenschaft und Verwaltungslehre, Karl-Franzens-Universität Graz

Ausgewählte Referenzgebiete europäischer Regulierung - Stand und Perspektiven Moderation

Prof. Dr. Ludwig GRAMLICH, Lehrstuhl für Öffentliches Recht und Öffentliches Wirtschaftsrecht, Technische Universität Chemnitz

\section{Finanzmärkte}

Institutionelle Aspekte der (neuen) Europäischen Finanzmarktregulierung und -aufsicht

Prof. Dr. Fabian AMTENBRINK, Professor of European Union Law, Erasmus Universität Rotterdam

Grundstrukturen der Regulierung der Finanzmärkte nach der Krise

Prof. Dr. Matthias LEHMANN, D.E.A., LL.M., Lehrstuhl für Bürgerliches Recht, Europäisches Privatrecht, Handels- und Wirtschaftsrecht, Internationales Privatrecht und Rechtsvergleichung, Martin-Luther-Universität Halle-Wittenberg

Die Rolle der EZB, des Eurosystems und des ESZB in der Finanzaufsicht unter Berücksichtigung des ESRB

Dr. Julian LANGNER, Zentralbereich Recht, Deutsche Bundesbank, Frankfurt am Main

1 Verordnung (EG) Nr. 1211/2009 des Europäischen Parlaments und des Rates vom 25. November 2009 zur Einrichtung des Gremiums Europäischer Regulierungsstellen für elektronische Kommunikation (GEREK) und des Büros, in: Amtsblatt der EU, Nr. L 337 vom 18. Dezember 2009, S. 1-10; Verordnung (EG) Nr. 713/2009 des Europäischen Parlaments und des Rates vom 13. Juli 2009 zur Gründung einer Agentur für die Zusammenarbeit der Energieregulierungsbehörden, in: Amtsblatt der EU, Nr. L 211 vom 14. August 2009, S. 1-14. 
kundärrechtliche Vorgabe mit Blick auf den europarechtlichen Anwendungsvorrang und die ultra vires-Lehre sowie anhand von Art. 23 Grundgesetz und dem Kerngehalt der nationalen Verfassungsidentität, die im Ergebnis allesamt nicht beeinträchtigt scheinen. Insofern könnten die regulierten Netzwirtschaften eine weitere Fallgruppe der im nationalen Verfassungsrecht anerkannten ,ministerialfreien Räume“ bilden. Schließlich wurde festgehalten, dass dem EU-Konzept zufolge eine Durchbrechung des Ministerialprinzips unter Legitimationsgesichtspunkten (nur) deshalb unbedenklich ist, weil es sich grundlegend vom deutschen Legitimationskonzept unterscheidet.

Die auf europäischer Ebene institutionalisierte Zusammenarbeit wurde sodann von Iris Henseler-Unger illustriert; als Referenzgebiete dienten die neuen europäischen Gremien im Bereich Telekommunikation (BEREC ${ }^{2}$ ) und Energie $\left(\mathrm{ACER}^{3}\right)$ sowie ein Überblick über den ,Recast ' des Eisenbahnpakets. Konstatiert wurde, dass die Herausforderungen in der europäischen, aber auch internationalen Perspektive einen Aus- und Umbau der Netze und damit erhebliche Investitionen erfordern. Nationale Besonderheiten rechtlicher wie tatsächlicher Art und das europäische Subsidiaritätsprinzip (Art. 5 Abs. 2 EU-Vertrag, EUV) sind dabei im Hinblick auf Netze und Märkte angemessen zu berücksichtigen. Die Regulierungsbalance zwischen Kommission, europäischen Regulierergruppen und nationalen Regulierungsbehörden muss allerdings fortlaufend geprüft und austariert werden.

Sodann plädierte Ulrich Stumpf für einen marktorientierten und wettbewerbsfördernden Regulierungsansatz mit einem Aufgreif(schwerwiegende Wettbewerbsprobleme), Eingreif- (Marktbeherrschung im Sinne des Wettbewerbsrechts) und Gestaltungskriterium für Regulierungseingriffe. Als Indikatoren für schwerwiegende Wettbewerbsprobleme stellte er einerseits marktbeherrschende Stel-
Basel III: Analyse der neuen Regeln

Prof. Dr. Michael SCHRÖDER, Leiter Forschungsbereich Internationale Finanzmärkte und Finanzmanagement, Zentrum für Europäische Wirtschaftsforschung, Mannheim, und Frankfurt School of Finance and Management, Frankfurt am Main

Diskussion und Zusammenfassung

Prof. Dr. Josef RUTHIG, Lehrstuhl für Öffentliches Recht, Europarecht, Rechtsvergleichung, Johannes-Gutenberg-Universität Mainz

Prof. Dr. Cornelia MANGER-NESTLER, LL.M., Professur für Deutsches und Internationales Wirtschaftsrecht, Hochschule für Technik, Wirtschaft und Kultur, Leipzig

\section{Wasserver- und Abwasserentsorgung}

Ausgewählte Referenzgebiete europäischer Regulierung - Wasserver- und Abwasserentsorgung

Prof. Dr. Mark OELMANN, Professur Infrastruktur und Netze, Hochschule Ruhr-West, Mühlheim an der Ruhr

\section{Telekommunikationsmärkte}

Telekommunikationsmärkte als Referenzgebiet europäischer Regulierung

Dr. Karl-Heinz NEUMANN, Direktor und Geschäftsführer, Wissenschaftliches Institut für Infrastruktur und Kommunikationsdienste, Bad Honnef

\section{Europäisierung der Regulierungspraxis}

RA Dr. Ernst Georg BERGER, SBR Rechtsanwälte, Düsseldorf

\section{Postdienste}

Postdienste - einschließlich eines Vergleichs zu Telekommunikation, Energie und Eisenbahn

Dr. Annegret GROEBEL, Abteilungsleiterin Internationales/Regulierung Post, Bundesnetzagentur, Bonn

Der Postsektor als Referenzgebiet europäischer Regulierungsbestrebungen

RA Dr. Stephan GERSTNER, Redeker Sellner Dahs, Rechtsanwälte, Berlin

\section{Verkehrsmärkte}

Verkehrsmärkte als Referenzgebiet europäischer Regulierungsbestrebungen - Stand und Perspektiven Prof. Dr. Hubertus GERSDORF, Gerd BuceriusStiftungsprofessur für Kommunikationsrecht und Öffentliches Recht, Universität Rostock

2 Body of European Regulators for Electronic Communications, mit Sitz in Riga (Lettland).

3 Agency for the Cooperation of Energy Regulators, mit Sitz in Ljubljana (Slowenien). 
lungen von Unternehmen (in der Telekommunikation auch ,beträchtliche Marktmacht'), wettbewerbshindernde Preise (,Verdrängungspreise'), andererseits aber auch schlechte Qualität heraus. Damit relevante Märkte für Regulierung in Betracht kommen, müssen wiederum drei Kriterien kumulativ erfüllt sein: 1. dauerhafte Marktschranken, 2. keine dynamische Tendenz zu Wettbewerb, die eine ex ante Regulierung redundant erscheinen lässt, 3. Insuffizienz des geltenden Wettbewerbsrechts, um diese Probleme zu lösen.

Schließlich wäre der Versuch einer sektorübergreifenden Betrachtung kein solcher, wenn Fragen des Rechtsschutzes auf nationaler wie supranationaler Ebene unbehandelt blieben. Die Rechtsprechungspraxis des Bundesverwaltungsgerichts, insbesondere das sogenannte Regulierungsermessen analysierte Kurt Graulich und widmete sich damit Entscheidungsprärogativen der nationalen Regulierungsbehörde im Spannungsfeld zu gerichtlicher Kontrolldichte. Um Strukturen des Rechtsschutzes gegen Maßnahmen von Regulierungsbehörden aufzuzeigen, wandte sich Joachim Scherer dem unionsrechtlichen Rahmen und dessen nationalstaatlicher Ausgestaltung zu. Dabei wurde auch deutlich, vor welchen Herausforderungen europäische beziehungsweise europäisierte prozessrechtliche Strukturen stehen. Den sektorübergreifenden Teil der Veranstaltung beendete Stefan Storr, der Fragen des Rechtsschutzes gegen Regulierungsmaßnahmen von Unionseinrichtungen in Österreich im sogenannten Beschwerdeverfahren sowie die Problematik der Rechtsverbindlichkeit von Unionsmaßnahmen in Regulierungsverbünden thematisierte.

Spezielle (Rechts-)Fragen ausgewählter Sektoren

Das von Ludwig Gramlich moderierte zweite Panel war ausgewählten Referenzgebieten gewidmet und sollte neben der Breite und Vielfalt der zu regulierenden beziehungsweise bereits regulierter Politikfelder zugleich die
Verkehrsmärkte als Referenzgebiet europäischer Regulierung

Prof. Dr. Karsten OTTE, Leiter Abteilung Eisenbahnregulierung, Bundesnetzagentur, Bonn

Markt- und Infrastrukturzugang im Flughafensektor - Eine ökonomische Analyse der europäischen Regulierungsvorgaben

Dr. Gernot MÜLLER, Abteilung Post, Logistik und Verkehr, Wissenschaftliches Institut für Infrastruktur und Kommunikationsdienste, Bad Honnef

\section{Energiemärkte}

Europäisierte Planung der Übertragungsnetze (TEN-E, NEPS, ENTSO-E, ACER)

Prof. Dr. Hartmut WEYER, Direktor des Instituts für deutsches und internationales Berg- und Energierecht, Technische Universität Clausthal

Regulierung der deutschen Energiewirtschaft, Historie und Entwicklungen

PD Dr. Christian GROWITSCH, Energiewirtschaftliches Institut (EWI) an der Universität zu Köln

Das Zusammenspiel von Regulierung und Wettbewerbskontrolle auf den Energiemärkten

Dr. Oliver KOCH, Generaldirektion Wettbewerb, Europäische Kommission, Brüssel

Diskussion und Zusammenfassung

Prof. Dr. Ludwig GRAMLICH, Lehrstuhl für Öffentliches Recht und Öffentliches Wirtschaftsrecht, Technische Universität Chemnitz

Suche nach einem gemeinsamen Kern befördern. Ausgehend vom jeweiligen Harmonisierungsniveau wurden sowohl institutionell-organisatorische Aspekte als auch der materiellrechtliche Stand der Regulierung erörtert.

\section{Finanzmärkte}

Obwohl die Finanzmärkte nicht zu den ,klassischen', weil netzbasierten Regulierungsfeldern zählen, erhellen andauernde Finanz- und Staatsschuldenkrisen, dass den regulatorischen Defiziten der Vergangenheit nur mit transnationalen Lösungsansätzen begegnet werden kann. Ausgehend davon, dass die Finanzmarktstabilität als gleichwertiges Ziel neben der Geldwertstabilität steht, sind das frühzeitige Erkennen und Eindämmen von 
systemischen Risiken der zentrale Auftrag einer ,prudential supervision and regulation“.

Ausgehend von institutionellen Aspekten der EU-Finanzmarktregulierung und -aufsicht befasste sich Fabian Amtenbrink mit dem neu gegründeten $\mathrm{ESFS}^{4}$, zusammengesetzt aus dem für die Makroaufsicht verantwortlichen $\mathrm{ESRB}^{5}$ und den für die Mikroaufsicht zuständigen EU-Behörden für Banken, Versicherungen und Wertpapiere, ${ }^{6}$ einem Gemeinsamen Ausschuss sowie den nationalen Aufsichtsbehörden. Dass der Netzverbundgedanke ein neues Integrationsniveau etabliert, steht außer Zweifel. Ob er die erhoffte und notwendige „präventive Wirkung“ erzielen wird, hängt nicht zuletzt von der mitgliedstaatlichen Akzeptanz seiner Beschlüsse $a b$.

Mit der Rolle von Zentralbanken bei der Aufsicht und Regulierung von Finanzmärkten beschäftigte sich Julian Langner und betonte mit Blick auf das Eurosystem, dass Reichweite und Grenzen des sogenannten makroprudentiellen Mandats der Europäischen wie nationalen Zentralbanken zukünftig noch weiter auszuloten seien.

Einen rechtsvergleichenden, transatlantischen Blick wagte Matthias Lehmann, indem er Grundstrukturen der Finanzmarktregulierung nach der Krise behandelte. Er betonte die Stärkung der internationalen und europäischen Ebene durch die Delegation von Regulierung auf Expertengremien sowie durch Zurückdrängung von Selbstregulierung. Gleichsam wurde die notwendige Reichweite der Regulierung dahingehend bestimmt, dass sowohl der Schattenbankensektor und die Ratingagenturen als auch OTC-Märkte ${ }^{7}$ mit einzubeziehen seien.
Schließlich unterzog Michael Schröder das reformierte Basel III-Regelwerk ${ }^{8}$ einer kritischen Bewertung; besonderes Augenmerk galt dem Aspekt, ob negative externe Effekte durch die starke Vernetzung der Banken über härtere und antizyklisch wirkende Eigenkapitalvorschriften aufgefangen werden können. Überdies stelle sich die Frage nach dem ,too big to fail“. Ob die vom Baseler Ausschuss festgesetzten Kernkapitalquoten letztlich greifen und antizyklisch wirken werden, kann nur vermutet werden. Nachdenklich stimmte die Erkenntnis, dass die Kennzahlen, zum Beispiel für Eigenkapitalpuffer, dem politischen Spektrum entspringen und ökonomisch derzeit nur schwer messbar scheinen.

\section{Wasserver- und Abwasserentsorgung}

Im Rahmen der Wasserver- und Abwasserentsorgung betrachtete Mark Oelmann unterschiedliche Ordnungsrahmen in Europa; besonders berücksichtigte er das Regulierungsmodell in England und Wales. Dabei wurden produktive, qualitative, dynamische und technische Effizienz als leitende Kriterien ebenso herausgestellt wie die Herangehensweise und der ökonomisch messbare Erfolg der Regulierung. In der Zukunftsperspektive sind England und Wales auf dem Weg zu einem Durchleitungswettbewerb, wobei alles darauf hindeutet, dass im englischen Wassersektor ein ähnliches Regulierungsverfahren wie in anderen Sektoren angewendet wird, mit ungewissen Erfolgsaussichten.

\section{Telekommunikationsmärkte}

Im Telekommunikationssektor untersuchte zunächst Karl-Heinz Neumann, inwiefern der europäische Ansatz zur Marktregulierung ein Erfolgsmodell ist und welche Konsequenzen

4 European System of Financial Supervision.

5 European Systemic Risk Board.

6 European Banking Authority (EBA), European Insurance and Occupational Pensions Authority (EIOPA) und European Securities Markets Authority (ESMA).

7 Als , Over-the-Counter-Märkte ‘ bezeichnet man außerbörslichen Handel zwischen Finanzmarktteilnehmern.

8 Als ,Baseler Accord' werden die - mehrfach reformierten (Basel II, III) - Empfehlungen des Basler Ausschusses für Bankenaufsicht bei der Bank für Internationalen Zahlungsausgleich (BIZ) zu Eigenkapitalanforderungen an Banken bezeichnet. 
der geänderte EU-Rechtsrahmen hat. Vorab wurde betont, dass europäische ,best practices" inzwischen auch vielen außereuropäischen Staaten als Referenzmodell dienen. Die vergleichsweise hohe Wettbewerbsintensität auf den Endkundenmärkten ist weitgehend ein Resultat der Regulierung der Vorleistungsmärkte. Der EU-Rechtsrahmen stellt eine graduelle Weiterentwicklung dar, wobei die Gefahr von Bürokratisierungstendenzen und unscharfer Ergebnisverantwortung aus der intensivierten Interaktion zwischen NRB, Europäischer Union und BEREC als möglicher Schwachpunkt ausgemacht wurde. Für den Übergang zu einer säkularen neuen hochleistungsfähigen Breitbandinfrastruktur wurden zwei Ansätze kritisch diskutiert: einerseits ein Universaldienst als anbieterfinanzierte interne Subventionierung, wobei die Vereinbarkeit mit EU-Recht strittig ist und andererseits die sogenannte 1Euro-Breitbandabgabe, gegen die gewichtige Verfassungsbedenken, fehlender Bedarf und administrativer Aufwand sprechen könnten.

Die praktischen Auswirkungen der Konsultations- und Notifizierungspflicht der BNetzA bei Entgeltverfahren (Art. 6, 7 Rahmenrichtlinie $^{9}$ ) analysierte Ernst-Georg Berger. Hierbei hat der EU-Rechtsrahmen 2009 die Einflussnahme durch die Kommission nochmals verstärkt. Insbesondere hat sich die Spruchpraxis der Beschlusskammern aufgrund eines durch die Kommission eingeleiteten Vertragsverletzungsverfahrens ${ }^{10}$ über den Umfang der Notifizierungspflicht geändert und es wird nunmehr mit der Einbeziehung der Entgeltregulierung eine europarechtskonforme Auslegung der $\S \S 12,13$ Telekommunikationsgesetz vorgenommen. ${ }^{11}$ Eine solche
Ausdehnung des Anwendungsbereichs auf Einzelfallentscheidungen erscheint im Ergebnis sachgerecht. Problematisch ist jedoch, dass der Entwurf des Telekommunikationsgesetzes 2011 keine Umsetzung der veränderten Spruchpraxis vorsieht. Letztlich sind die Auswirkungen der Konsultations- und Notifizierungspflicht auf die Spruchpraxis durch längere, unsichere Verfahren und eine verstärkte Lobbyarbeit sowie politischen Einfluss erheblich.

\section{Postdienste}

Auf dem Sektor für Postdienste konnten durch die vollständige Marktöffnung 2011 ${ }^{12}$ in fast allen , alten' Mitgliedstaaten Chancen für eine Wettbewerbsintensivierung geschaffen werden, konstatierte Annegret Groebel. $\mathrm{Zu}$ einem „regulatory level playing field“ und zur Entwicklung von europäischen ,best practices' wird die ERGP ${ }^{13}$ beitragen; besonders wertvoll sind dabei Erfahrungen, die NRB mit bereits länger vollständig geöffneten Märkten in die ERGP einbringen. Entsprechend dem „EU-Trend“ in den Bereichen Telekommunikation, Energie und Eisenbahn ist auch hier eine Tendenz zu neuen Agenturen, zunehmender Institutionalisierung und mithin steigendem Einfluss der Europäischen Union auf nationale Regulierung zu beobachten. Gleichzeitig wurden jedoch die Gefahren einer Vermischung beziehungsweise Verlagerung von Kompetenzen unter dem Gesichtspunkt des Subsidiaritätsprinzips und einer ausufernden Bürokratisierung deutlich gemacht.

Als mögliche Referenzpunkte für einen EURegulierungsrahmen im Postsektor benannte

9 Richtlinie 2008/6/EG des Europäischen Parlaments und des Rates vom 20. Februar 2008 zur Änderung der Richtlinie 97/67/EG im Hinblick auf die Vollendung des Binnenmarktes der Postdienste der Gemeinschaft, in: Amtsblatt der EU, Nr. L 108 vom 24. April 2002, S. 33-50.

10 Art. 258 Vertrag über die Arbeitsweise der Europäischen Union (AEUV).

11 Vgl. zum Beispiel Bundesnetzagentur, Beschlusskammer 3: Beschluss in dem Verwaltungsverfahren aufgrund des Antrages der E-Plus Mobilfunk GmbH \& Co. KG vom 21.09.2010 wegen Genehmigung der Entgelte für Terminierungsleistungen im Mobilfunknetz der Antragstellerin und damit in Zusammenhang stehender weiterer Leistungsentgelte, BK 3a-10/101 vom 28.10.2010.

12 Richtlinie 2008/6/EG im Hinblick auf die Vollendung des Binnenmarktes, 2008.

13 European Regulators Group for Postal Services. 
Stefan Gerstner eine erhöhte regulatorische Effektivität durch einen Infrastrukturzugang ohne Entflechtung sowie die Tatsache, dass keine Alternative zwischen der Effizienz der Infrastrukturbereitstellung und der Finanzierung nicht wettbewerbsüblicher Kosten bestehen. Dass Qualitätsregulierung durch Regulierung der nachgelagerten Märkte bedingt ist, aber zugleich Infrastrukturgewährleistung und Verhaltenskontrolle auf dem vorgelagerten Markt erfordert, wurde als weiterer Referenzpunkt eines europäisierten Regulierungsrahmens im Postsektor benannt.

\section{Verkehrsmärkte}

Hubertus Gersdorf betonte, dass sich die Verkehrsmärkte stärker als andere Sektoren in einem inter- und intramodalen Wettbewerb befinden. Der Güter- und Personenverkehr ist maßgeblich durch Schiene, Straße und Luft geprägt. Die Regulierung verfolgt hier das Ziel eines einheitlichen europäischen Verkehrsraums mit einem wettbewerbsorientierten und ressourcenschonenden Verkehrssystem. ${ }^{14}$ Auf der anderen Seite verfolgt die Europäische Union in den nächsten Jahren ehrgeizige umweltpolitische Ziele (Reduzierung der $\mathrm{CO}_{2}$-Emissionen), manifestiert unter anderem durch Verlagerung des Güter- sowie eines Großteils des Personenverkehrs von der Straße auf die Schiene. Allerdings ist keinerlei Harmonisierung bei der Internalisierung externer Kosten ${ }^{15}$ zu verzeichnen und auch kein vergleichbares Regulierungsniveau wie in anderen Bereichen vorhanden.
Für den Eisenbahnsektor zeigte Karsten Otte auf, welche möglichen Auswirkungen die EU-Verordnung zur Schaffung eines europäischen Schienennetzes für einen wettbewerbsfähigen Güterverkehr ${ }^{16}$ auf dem deutschen Schienenverkehrsmarkt haben wird; besonders thematisierte er den Gesichtspunkt der Veränderung des Stellenwertes des Güterverkehrs gegenüber dem Personenverkehr.

Eine ökonomische Analyse der europäischen Regulierungsvorgaben beim Markt- und Infrastrukturzugang im Flughafensektor präsentierte Gernot Müller. Eingehend behandelt und kritisch hinterfragt wurden dabei Verfahrensregeln für die Zuweisung von Zeitnischen ${ }^{17}$, die Festlegung und Kontrolle von Flughafenentgelten ${ }^{18}$ sowie die Liberalisierung der Erbringung von Bodenabfertigungsdiensten ${ }^{19}$.

\section{Energiemärkte}

Die große Bedeutung der erneuerbaren Energien infolge hoher Zuwachsraten bei Solarund Windenergie leitete die Diskussion im Rahmen der Energiemärkte ein. Wie Christian Growitsch deutlich machte, erfordert der enorme Leistungszuwachs einen Leitungsausbau von Norden nach Süden, wobei der nationale Fokus auf dem Süden Ostdeutschlands Richtung Mitteldeutschland liegt. Der Zubau auf allen Netzebenen ist wohl die am leichtesten umsetzbare politische Handlungsalternative nach dem AKW-Moratorium, obgleich damit Fragen der Netzstabilität und Systemsicherheit nicht gelöst sind. Mit der Entwick-

14 Vgl. Europäische Kommission: Weißbuch. Fahrplan zu einem einheitlichen europäischen Verkehrsraum - Hin zu einem wettbewerbsorientierten und ressourcenschonenden Verkehrssystem, KOM (2011) 144.

15 Kosten, die von Verkehrsteilnehmern verursacht, aber nicht von ihnen getragen werden, zum Beispiel Stau, Unfälle, Lärm.

16 Verordnung (EU) Nr. 913/2010 des Europäischen Parlaments und des Rates vom 22. September 2010 zur Schaffung eines europäischen Schienennetzes für einen wettbewerbsfähigen Güterverkehr, in: Amtsblatt der EU, Nr. L 276 vom 20. Oktober 2010, S. 22-32.

17 Verordnung (EWG) Nr. 95/93 des Rates vom 18. Januar 1993 über gemeinsame Regeln für die Zuweisung von Zeitnischen auf Flughäfen in der Gemeinschaft, in: Amtsblatt der EU, Nr. L 14 vom 22. Januar 1993, S. 1-6.

18 Richtlinie 2009/12/EG des Europäischen Parlaments und des Rates vom 11. März 2009 über Flughafenentgelte, in: Amtsblatt der EU, Nr. L 70 vom 14. März 2009, S. 1-6.

19 Richtlinie 96/67 EG des Rates vom 15. Oktober 1996 über den Zugang zum Markt der Bodenabfertigungsdienste auf den Flughäfen der Gemeinschaft, in: Amtsblatt der EU, Nr. L 272 vom 25. Oktober 1996, S. 36-45. 
lung neuer Technologien besteht aber eine deutliche Optimierungsperspektive.

Mit Blick auf die europäisierte Planung der Übertragungsnetze stellte Hartmut Weyer klar, dass die Ziele der Netzplanung hier klar definiert sind: Versorgungssicherheit, Umweltverträglichkeit und Wettbewerbsförderung. Die Prüfung und Beobachtung der Umsetzung von Netzentwicklungsplänen obliegt ACER. Zugleich wurde klargestellt, dass TEN-E ${ }^{20}$ Vorhabenpläne nicht die gewünschte Wirkung entfaltet haben. Gleichwohl sind Netzentwicklungspläne auf nationaler Ebene die besseren Instrumente der Bedarfsplanung. Nicht unbeachtet blieb auch der Referentenentwurf des Energiewirtschaftsgesetzes 2011, der deutlich mehr Markt und Wettbewerb durch stärkere Entflechtungsregeln fördert.

Abschließend illustrierte Oliver Koch das Zusammenspiel von europäischer Regulierung und EU-Wettbewerbskontrolle und bekräftigte den fortschreitenden Einfluss der europäischen Ebene. Zugleich wurde betont, dass sich abstrakt-generelle Regulierung und einzelfallorientierte Wettbewerbskontrolle nicht gegenseitig ausschließen, sondern aus europäischer Sicht als komplementäre Elemente einer umfassenden Marktgewährleistung zu verstehen sind. Diese Art der Kooperation zwischen Regulierungs- und Wettbewerbsbehörden ist gleichsam für ein kohärentes Agieren unverzichtbar.
Konsequenzen für den Infrastrukturwettbewerb der Zukunft

Vorerst bleibt offen, ob die erkennbaren Gemeinsamkeiten zwischen den Regulierungsfeldern größer sind als die vorhandenen sektorspezifischen Unterschiede und insofern einer größeren Systembildung entgegenstehen. Festzuhalten ist jedoch die zunehmende Komplexität und Dynamik der Materie sichtbar an notwendiger, aber zeit- und kostenintensiver Koordinierung und Implementierung europäischer wie internationaler Vorgaben. Darüber hinaus haben sich signifikante sektorspezifische und auch nationale Besonderheiten herausgebildet, die es angemessen zu berücksichtigen gilt und die nicht ohne Weiteres im Wege der, ,regulierungsrechtlichen Vollharmonisierung" umgesetzt werden können. Im Rahmen der immer engeren Verflechtung im europäischen Verbund zeigt sich bereits heute ein ausgewogenes Bild der Kooperation im Mehrebenensystem, wobei im Zweifel der Europäischen Kommission die Rolle als ,regulierungsrechtliche Drehscheibe“" zukommt.

Schließlich müssen mit einer europäisierten beziehungsweise internationalisierten Rechtund Regelsetzung notwendig einhergehende, neuartige Fragen demokratischer Legitimation und Partizipation ernst genommen und verträglichen Lösungen zugeführt werden.

20 Transeuropäische Energienetze. 